\title{
Facial Age Group Classification
}

\author{
Jignesh Prajapati, Ankit Patel, Punit Raninga \\ EC Department, Parul Institute of Engineering \& Technology, Limda, Vadodara, India
}

\begin{abstract}
Estimating human age group automatically via facial image analysis has lots of potential real-world applications, such as human computer interaction and multimedia communication. However, It is still a challenging problem for the existing computer vision systems to automatically and effectively estimate human age group. The aging process is determined by not only the person's gene, but also many external factors, such as health, living style, living location, and weather conditions. Males and females may also age differently. An age group classification system for facial images is proposed in this paper. Five age groups including babies, children, young adults, middle-aged adults, and old adults, are used in the classification system. The process of the system is divided into threephases: location, feature extraction, and age classification. Geometric features are used to distinguish whether the face is baby or child. Wrinkle features are used to classify the image into one of three adult groups- young adults, middle-aged adults, and old adults.
\end{abstract}

Key Words: Age Classification, Facial Feature Extraction.

\section{Introduction}

The human traits displayed by facial attributes, such as personal identity, facial expression, gender, age, ethnic origin, and pose, have attracted much attention in the last several decades from both industry and academic since face image processing techniques yield extensive applications in graphics and computer vision fields. However, the variation is often inevitable due to the generic difference between different individuals and environmental/ artificial factors.

In this paper, first the facial image is converted into gray scale image. Then location phase is used to detect the face, and then compute the location of eyes, nose and mouth. Then various distances and ratios between the geometric features (eyes, nose and mouth) are computed. These calculations are used to distinguish whether the face is baby or child. Then winkles from the face are extracted. Wrinkle features are used to classify the image into one of three adult groups- young adults, middle-aged adults, and old adults. The facial image is classified into Five age groups, including babies, children, young adults, middle-aged adults, and old adults.

TABLE 1 AGE GROUP NAME WITH ITS AGE RANGE

\begin{tabular}{|c|c|}
\hline Age Group Name & Age Ranges \\
\hline Babies & $0-2$ \\
\hline Children & $3-16$ \\
\hline Young Adults & $17-30$ \\
\hline Middle-aged Adults & $31-45$ \\
\hline Old Adults & Above 45 \\
\hline
\end{tabular}

The experiments are performed over FG-NET[6] database. The FG-NET aging database is publicly available. It contains 1,002 high-resolution color or gray-scale face images of 82 multiple-race subjects with large variation of lighting, pose, and expression. The age range is from 0 to 69 years with chronological aging images available for each subject (on average, 12 images per subject).

\subsection{Real-World Applications}

There are many popular real-world applications related to age synthesis and estimation [1].

\section{- Forensic Art:}

Age progression is used to modify and enhance photographs by computer or manually (with professional hand drawing skills) for the purpose of suspect/victim and lost person identification with law enforcement.

When the photos of missing family members (especially children) or wanted fugitives are outdated, forensic artists can predict the natural aging of the subject faces and produce updated face images, utilizing all available individual information, such as facial attributes, lifestyle, occupation, and genetics.

Age synthesis by machine can significantly enhance the efficiency of the forensic artist while at the same time providing more photorealistic aging/ rejuvenating effects that can satisfy the needs of aesthetics. 


\section{- Electronic Customer Relationship Management (ECRM)}

The ECRM is a management strategy to use information technology and multimedia interaction tools for effectively managing differentiated relationships with all customers and communicating with them individually. Since different groups of customers have very different consuming habits, preferences, responsiveness, and expectation to marketing, companies can gain more profits by acknowledging this fact, responding directly to all customers' specific needs, and providing customized products or services. the most challenging part hereby is to obtain and analyze enough personal information from all customer groups, which needs companies to establish long-term customer relationships and sustain a large amount of cost input.

\section{- Security Control and Surveillance Monitoring:}

With the input of a monitoring camera, an age estimation system can warn or stop underage drinkers from entering bars or wine shops; prevent minors from purchasing tobacco products from vending machines; refuse the aged when he/she wants to try a roller coaster in an amusement park; and deny children access to adult Web sites or restricted movies.

\section{- Biometrics:}

In real face recognition or identification applications, it is often the case that the system needs to recognize or identify faces after a gap of several years such as passport renewal and border security, which reveals the importance of age synthesis.

\section{- Entertainment:}

Without any noticeable artifacts in many such movies, the actor's appearance can be transformed from young to old or reverse instantly or gradually with extremely realistic aging effects. Some of these mysterious visual effects are generated by age synthesis techniques to provide fantastic experiences to audiences.

\section{- Cosmetology:}

People want to have their faces look younger. Rejuvenation, implemented by cosmetic surgery (shape) or skin care (texture), is a popular beauty treatment in cosmetology. Computer-aided age synthesis is obviously a useful technique to predict the rejuvenating results as references that can help beauticians or surgeons in the treatments and operations. With a face rejuvenating system available, more individual-oriented services can be provided to patients, who can directly see the computer synthetic photorealistic results and make changes with their preferences beforehand.

\subsection{HUMAN AGING ON FACES}

Human face aging is generally a slow and irreversible process, even though some retinoids (e.g., tretinoin) may slightly reverse minor photoaging effects. Although people are aging differently and aging shows different forms in different ages, there are still some general changes and resemblances we can always describe. From the biological or anthropometric point of view, there are roughly two stages during the human life that are quite different in face growth, development, and aging forms.

1. craniofacial aging (shape change)

2. adult aging

\subsubsection{Craniofacialaging (shape change)}

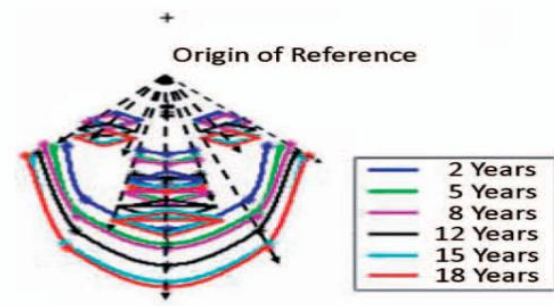

Figure 1 Craniofacial growth model for age progression[1]

During the early growth and development of the face, from birth to adulthood, the greatest change is the craniofacial growth (shape change) [1]. Overall, the face size is getting larger gradually during the craniofacial growth. Fig. 3d shows six prototype faces, originally shown in, with craniofacial growth from 2 to 18 years, 3 years per sketch. The shape synthesis is based on the anthropometric measurements with the growth of the cranium, the forehead slopes back, shrinks, and releases spaces on the surface of the cranium, while the facial 
features, such as eyes, nose, ears, and mouth, expand their areas and tend to cover these interstitial spaces. Cheeks extend to larger areas and the chin becomes more protrusive. The facial skin relatively does not change too much compared with the craniofacial growth. But facial hairs, such as a mustache, may become dense and even bushy. Skin color may change a little bit.

\subsubsection{Adult aging}

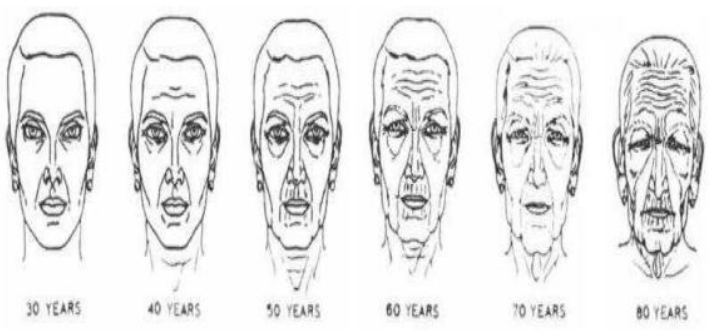

Figure 2. Face aging sketches from 30 to 80 years with 10 years per sketch[1]

During adult aging, from adulthood to old age, the most perceptible change becomes skin aging (texture change). The shape change still continues, but less dramatically, mostly due to typical patterns in skin and tissue. Fig. 2 shows six face aging sketches from 30 to 80 years, with 10 years per sketch. Biologically the face matures and ages with loss of collagen beneath skin as well as gravity effects, the skin becomes thinner, darker, less elastic, and more leathery. A dynamic wrinkles and blemishes due to biologic aging gradually appear. Dynamic wrinkles and folds due to muscle motion become more distinct. In the areas of deeper attachment, such as cheeks, eyelids, chin, and nose, elasticity of muscles and soft tissues gets weak and fat continues depositing. In other areas, fat may atrophy or be absorbed. These changes lead to the downward descent or sagging ofthe skin, such as double chin, dropping cheek, and lower eyelid bags. Although the craniofacial growth is not dramatic during this aging period, the facial geometry change is still evident from 30 to 80 years, especially in the female faces. Faces change from a U-shaped to a trapezoid or rectangle. The bony framework underneath the skin may also deteriorate to accelerate the development of skin aging, such as wrinkles, creases, and droops. In addition, face aging during this age period may cause the loss of flexible control of facial muscles so that the facial movements and behaviors may also change unintentionally.

\section{The Proposed System}

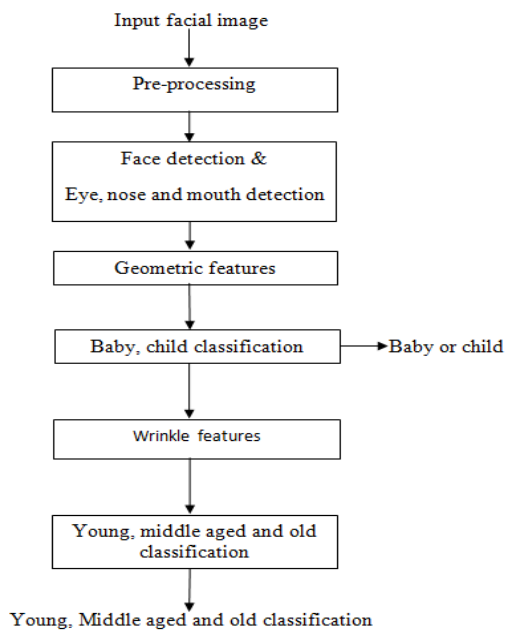

Figure 3. Proposed system

\subsection{Pre-processing}

In order to make the feature extraction step easier, a set of functions to pre-process the the input image has been developed. The pre-processing step require following steps.

\subsubsection{RGB ToGray:}

The first step is to convert the input images from RGB to gray space. The transformation from RGB to grey space is done using the eqation

$$
\mathrm{Y}=0.3 \mathrm{R}+0.59 \mathrm{G}+0.11 \mathrm{~B}
$$


But we do not need to make our implementation because we can use the Matlab function Y_image $=$ rgb2gray $\left(R G B \_i m a g e\right)$.

\subsubsection{Crop}

It is frequently the case that the input image has more information than just the face, so the background and part of the body also appear in the image. Since we will only use facial information we need the image to be fitted to the face due to avoid this extra information than will only affect the system performance.

\subsubsection{Resize}

The amount of features that returns some feature extraction functions depend on the number of pixels in the image. Due to the fact that we need to extract the same amount of features from each input sample, all the image have to be resized to fixed size.

Like in crop, the image size is concern point in the project develop thereby initial values are set up. The initial values are 256 pixels in height and 192 pixels in width. The resize is done with the Matlab function im_r=imresize(im,[256,192]) which allows as an input the original image and the size of new one, and returns as an output the resized image.

\section{3 face detection \& eye, nose and mouth detection}

detector $=$ vision.CascadeObjectDetectorcreatesa System object, detector, that detects objects using the Viola-Jones algorithm. The Classification Model property controls the type of object to detect. By default, the detector is configured to detect faces.

detector $=$ vision .CascadeObjectDetector(MODEL) creates a System object, detector, configured to detect objects defined by the input string, MODEL. The MODEL input describes the type of object to detect. There are several valid MODEL strings,such as 'FrontalFaceCART', 'UpperBody',and 'ProfileFace'. See the Classification Model property description for a full list of available models.

\subsubsection{Face detection}

FrontalFaceCART (Default):Detects faces that are upright and forward facing. This model is composed of weak classifiers, based on the classification and regression tree analysis (CART). These classifiers use Haar features to encode facial features. CART-based classifiers provide the ability to model higher-order dependencies between facial features.

\subsection{2 eyes detection:}

EyePairBig or EyePairSmall: Detects a pair of eyes. The EyePairSmall model is trained using a smaller image. This enables the model to detect smaller eyes than the EyePairBig model can detect.

\subsubsection{Nose detection:}

Nose: This model is composed of weak classifiers, based on a decision stump, which use Haar features to encode nose details.

\subsubsection{Mouth detection:}

Mouth: Detects the mouth. This model is composed of weak classifiers, based on a decision stump, which use Haar features to encode mouth details.

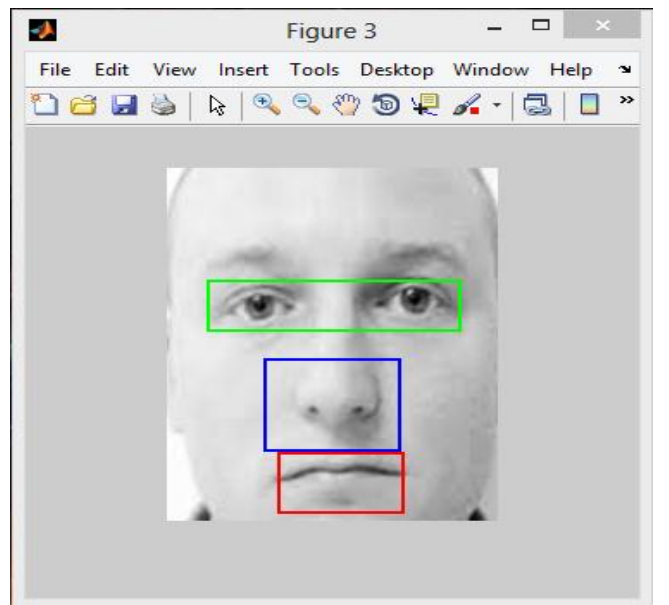

Figure 4.face detection and eyes, nose and mouth detection 


\subsection{Geometric features:}

Geometric features are used for aging detail in craniofacial growth. In this phase different ratios are obtained as shown in figure below. Geometric features are obtained using location of eyes, nose and mouth.
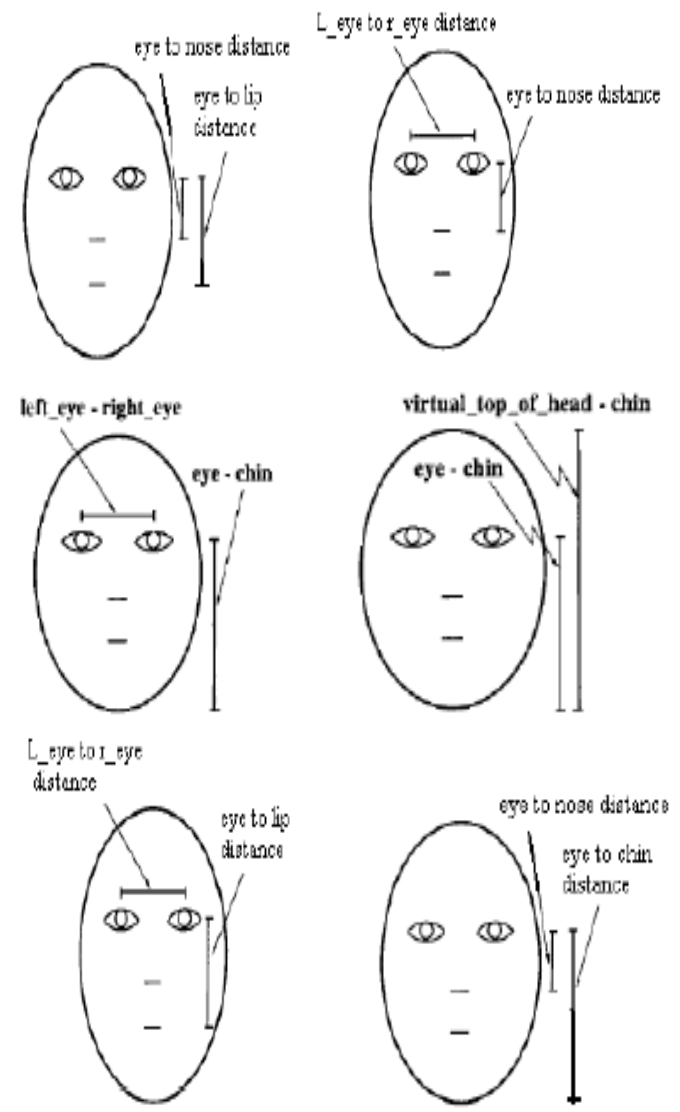

Figure 5 various geo-metric ratios[5]

Using these ratios the threshold is determined which is used to differentiate baby and child.

\subsection{Wrinkle features}

For differentiate young, middle aged and old, we extract winkle features. Here gradient operation is used for wrinkle detection.

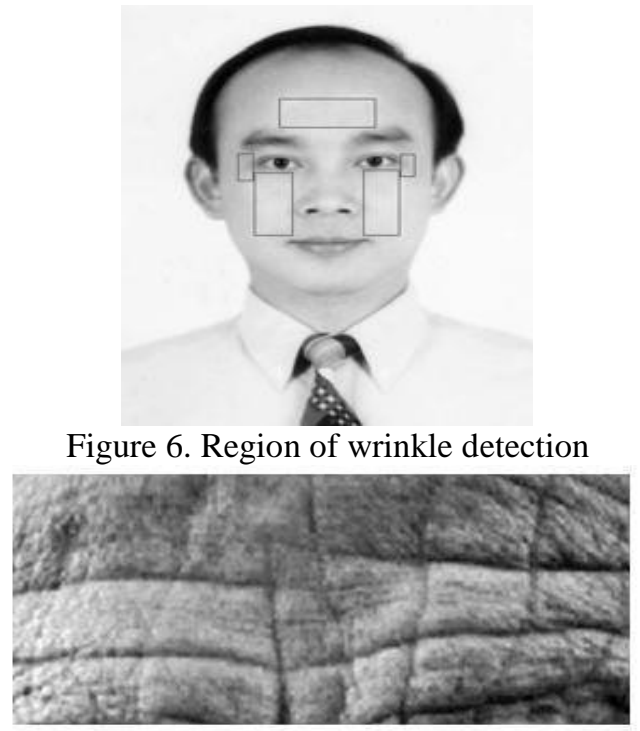

(a) 


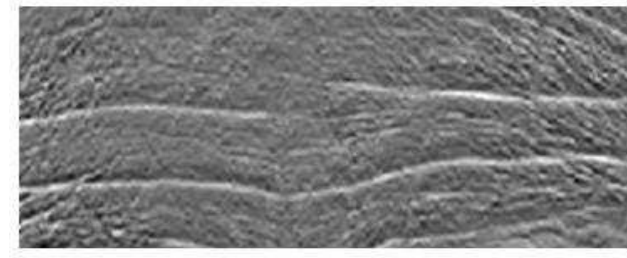

(b)

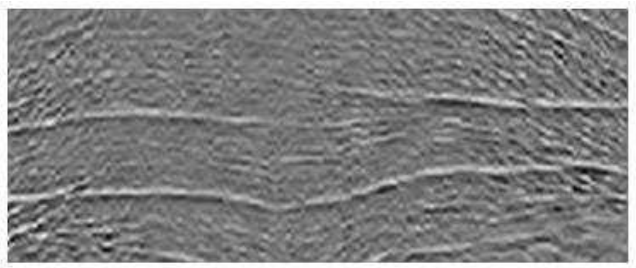

(c)

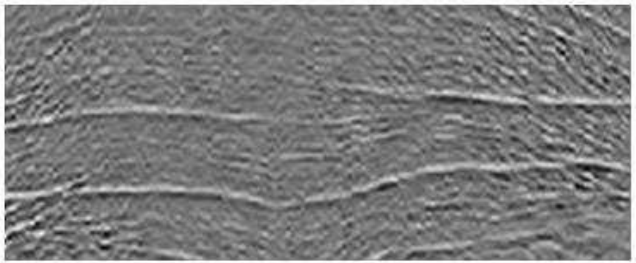

(d)

Figure 7.wrinkle detection using gradient

(a) Input face (b) first gradient (c) second gradient (d) binarized image with threshold 0.03.

Wrinkle density is used to classify young, middle aged and old faces. Wrinkle density is given by

$$
\text { Density }=\frac{\text { Wrinkle pixels }}{\text { Total pixels }}
$$

By adjusting appropriate threshold, we can classify young, middle aged and old faces.

\section{Experimental Results}

The experiment I performed over FG-net database to classify the facial image into five age groupsbaby, child, young, middle aged and old. The input face and output results are shown below.

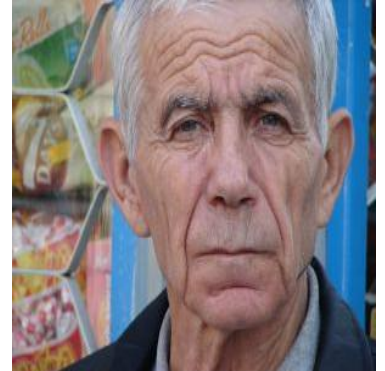

(a)

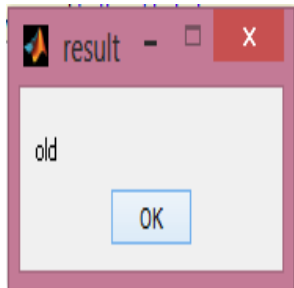

(b)

Figure 8. (a) Input image (b) Output result.

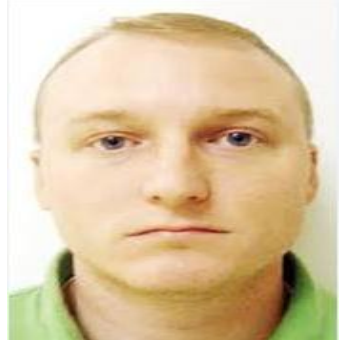

(a)

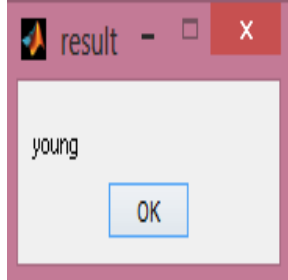

(b)

Figure 9. (a) Input image (b) Output result. 


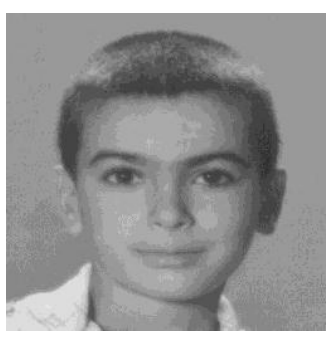

(a)

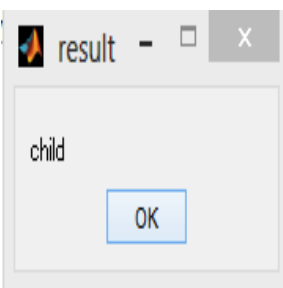

(b)

Figure 10. (a) Input image (b) Output result.

\section{Conclusion And Future Work}

In this paper age is classified into five groups- baby, child, young, middle aged and old using geometric and wrinkle features. Geometric features are used to distinguish whether the face is baby or child. Wrinkle features are used to classify the image into one of three adult groups- young adults, middle-aged adults, and old adults.

In future work efforts will be applied to estimate the human age from the facial image.

\section{References}

\section{Papers:}

[1]. Yun Fu, Thomas S. Huang, "Age Synthesis and Estimation via Faces: A Survey”IEEE transactions on pattern analysis and machine intelligence, November 2010

[2]. Sarah N. Kohail, "Using Artificial Neural Network for Human Age Estimation Based on Facial Images" 2012 International Conference on Innovations in Information Technology, 2012@IEEE.

[3]. AlirezaKeshavarzChoobeh "Improving Automatic Age Estimation Algorithms using an Efficient Ensemble Technique" International Journal of Machine Learning and Computing,April 2012@ IEEE.

[4]. Li liu, Jianminng Liu, Jan cheng. "Age-Group classification of facial image"International Journal of Machine Learning and application, 2012

[5]. Petra GRD, "Introduction to human age estimationusing face images", faculty of materials science and technology in trnavaslovak university of technology in Bratislava,2013

Websites:

[6]. The FG-NET Aging Database, http://www.fgnet.rsunit.com 